\title{
Relative Entropy in 2D Quantum Field Theory, Finite-Size Corrections, and Irreversibility of the Renormalization Group
}

\author{
José Gaite \\ Instituto de Matemáticas y Física Fundamental, CSIC, Serrano 123, 28006 Madrid, Spain
}

(Received 3 November 1997)

\begin{abstract}
The relative entropy in two-dimensional field theory is studied for its application as an irreversible quantity under the renormalization group, relying on a general monotonicity theorem for that quantity previously established. In the cylinder geometry, interpreted as finite-temperature field theory, one can define from the relative entropy a monotonic quantity similar to Zamolodchikov's $c$ function. On the other hand, the one-dimensional quantum thermodynamic entropy also leads to a monotonic quantity, with different properties. The relation of thermodynamic quantities with the complex components of the stress tensor is also established and hence the entropic $c$ theorems are proposed as analogs of Zamolodchikov's $c$ theorem for the cylinder geometry. [S0031-9007(98)07422-5]
\end{abstract}

PACS numbers: 11.10.Gh, 05.70.Jk, 11.10.Kk

In Euclidean quantum field theory (QFT) it is possible to define a type of entropy, the relative entropy, which is a monotonic function of the couplings and increases in the crossover from one multicritical point to another of lower order [1]. Therefore, it is a suitable quantity to embody the irreversibility of the action of the renormalization group (RG). There is also a well-known and celebrated monotonic quantity in two-dimensional (2D) QFT, Zamolodchikov's $c$ function [2]. Although a priori there is no connection between both quantities, some arguments indicate that such a connection may nevertheless exist. For example, Zamolodchikov's $c$ function is supposed to count the independent degrees of freedom in a model near the critical point (CP). This certainly agrees with the statistical definition of entropy. On the other hand, the central charge of 2D conformal field theory (CFT), to which Zamolodchikov's $c$ function reduces at the $\mathrm{CP}$, has been shown to coincide with a particular type of renormalized entropy, the geometric entropy [3]. These arguments beg for an investigation on whether a relation between Zamolodchikov's $c$ function and some type of entropy in 2D exists off the CP.

We study here the properties of the relative entropy in general 2D models, in regard to its connection with Zamolodchikov's $c$ function, with explicit computations for the Gaussian and the Ising models. We shall always consider continuum theories with UV cutoff $\Lambda$ and we shall further introduce an IR cutoff, for example, by giving the system a finite size. A particular finite geometry has an interesting interpretation: The classical partition function in the strip or cylinder of width $\beta$ is equivalent to the one-dimensional quantum partition function at temperature $T=1 / \beta$. We can then calculate thermodynamic functions of this quantum system, for example, the quantum specific entropy. This entropy will also have a role as a monotonic quantity.

The idea of applying finite-size scaling methods to Zamolodchikov's theorem appeared in [4,5]. The form of the finite-size corrections to the free energy for the Gaussian model and the Ising model off their CP has been obtained in [6]. There it is briefly discussed their connection with Zamolodchikov's $c$ function, concluding that they differ but making no further analysis. The compact dimension $\beta$ can be used as RG parameter, providing a thermodynamic interpretation of the RG $[7,8]$. The finitesize correction to the free energy was used as a candidate monotonic function in [7], concluding that a thermodynamic analog of Zamolodchikov's theorem holds for it but only under an additional condition which cannot be deduced from thermodynamics rules. Here, relying on the monotonicity theorem for the relative entropy [1] we shall propose a monotonicity theorem analogous to Zamolodchikov's. We shall further prove a new thermodynamic monotonicity theorem involving the quantum specific entropy.

Given the cutoff logarithm of the partition function per unit volume $W[\lambda, \Lambda]$, the relative entropy is the Legendre transform of $W[\lambda, \Lambda]-W[0, \Lambda]$ with respect to the relevant couplings $\lambda_{a}$,

$$
S_{\text {rel }}=W-W_{0}-\lambda^{a} \partial_{a} W .
$$

Now we select one coupling $\lambda$ - or take a common factor of all the couplings - to evaluate the change of $S_{\text {rel }}$ with respect to it. To be precise, we must use the difference between the couplings and their critical values, since the $\mathrm{CP}$ is taken as the reference for the relative entropy. (The critical couplings may be null in some cases.) We have the following general monotonicity theorem [1]

$$
\lambda \frac{d S_{\mathrm{rel}}}{d \lambda}=\left\langle\left(I_{\lambda}-\left\langle I_{\lambda}\right\rangle\right)^{2}\right\rangle \geq 0,
$$

where $I_{\lambda}=\lambda \int \Phi$ is the relevant part of the action containing the coupling that we consider. Let us introduce the stress tensor trace, $\Theta:=T_{a}^{a}$, which in general is proportional to the relevant part of the action; more precisely,

$$
\Theta=\lambda y \Phi, \quad \text { with } y=2-d_{\Phi}>0,
$$


where $d_{\Phi}$ is the scaling dimension of the field $\Phi$. Hence, we can write the monotonicity theorem as

$$
\begin{aligned}
\lambda \frac{\partial S_{\text {rel }}}{\partial \lambda} & =\frac{1}{y^{2}} \int d^{2} z\langle[\Theta(z)-\langle\Theta(0)\rangle][\Theta(0)-\langle\Theta(0)\rangle]\rangle \\
& \geq 0 .
\end{aligned}
$$

We would like to derive a general expression for $S_{\text {rel }}$. We can use the scaling form

$$
W(\lambda, \Lambda)=\Lambda^{2} \mathcal{F}\left(\frac{\lambda^{2 / y}}{\Lambda^{2}}\right) .
$$

In the absence of logarithmic corrections, $\mathcal{F}$ is an analytic function [9], so $W$ can be expanded as

$$
W(\lambda, \Lambda)=\Lambda^{2} F_{0}+F_{1} \lambda^{2 / y}+O\left(\Lambda^{-2}\right) .
$$

The UV divergent term is irrelevant for the relative entropy and in the infinite cutoff limit

$$
S_{\text {rel }}(\lambda)=W(\lambda)-W(0)-\lambda \frac{d W}{d \lambda}=F_{1} \frac{y-2}{y} \lambda^{2 / y} .
$$

Taking into account that $y<2$ and $F_{1}<0$ we have that $S_{\text {rel }}(\lambda)>0$ and it increases with $|\lambda|$. However, given the simple scaling form of $S_{\text {rel }}(\lambda)$, this statement is not very informative. We will obtain a more illuminating version when we introduce a finite geometry.

Let us now consider solvable models, namely, the Gaussian model $\left(d_{\Phi}=0\right)$ and the Ising model $\left(d_{\Phi}=1\right)$, which, on the other hand, exhibit logarithmic corrections. The relative entropy per unit volume of the Gaussian model calculated using dimensional regularization was given in [1]. It can be expressed as

$$
S=\frac{\Gamma[(4-d) / 2]}{(4 \pi)^{d / 2} d} t^{d / 2},
$$

which in $d=2$ yields

$$
S=\frac{t}{8 \pi},
$$

with $t=r-r_{c}=r \equiv m^{2}, m$ being the mass parameter.

It is more illustrative to start with the expression of the cutoff logarithm of the partition function per unit volume

$$
W(r) \equiv-\ln Z= \pm \frac{1}{2} \int_{0}^{\Lambda} \frac{d^{2} p}{(2 \pi)^{2}} \ln \frac{p^{2}+r}{\Lambda^{2}}
$$

for free bosons (upper sign) or Majorana fermions (lower sign). It can be integrated exactly and yields

$$
W(r)= \pm \frac{1}{8 \pi}\left[-\Lambda^{2}+r \ln \frac{\Lambda^{2}}{r}+r+O\left(\Lambda^{-2}\right)\right],
$$

exhibiting a quadratic and a logarithmic divergence. We have in the infinite cutoff limit

$$
S_{\text {rel }}(r)=W(r)-W(0)-r \frac{d W}{d r}=\frac{r}{8 \pi},
$$

for the Gaussian model, in accord with the dimensional regularization result. For the Ising model,

$$
\begin{aligned}
S_{\text {rel }}(r) & =W(r)-W(0)-m \frac{d W}{d m} \\
& =-\frac{m^{2}}{8 \pi}\left(1+\ln \frac{m^{2}}{\Lambda^{2}}\right) .
\end{aligned}
$$

The relative entropy is monotonic with $\mathrm{m}^{2}$ for both models. However, the presence of the logarithmic correction in the latter case signals that it is not well defined in the continuum limit $\Lambda \rightarrow \infty$ unless we introduce a renormalization scale or, alternatively, an IR cutoff. This is the general situation for models with logarithmic corrections.

Let us now consider a finite-size geometry, in particular, a cylinder, equivalent to finite temperature field theory. It provides an IR cutoff with physical interest. The partition function is $Z=\operatorname{Tr} e^{-\beta H}$, which can be represented as a functional integral on $S^{1} \times \mathbb{R}$ with $\beta=1 / T$ the length of the compact dimension. The specific logarithm of the partition function on a cylinder of width $\beta$ and length $L$ has a finite-size expression as $L \rightarrow \infty$ :

$$
\frac{-\ln Z}{L}=\beta \frac{F}{L}=e_{0}(\Lambda, m) \beta+\frac{C(\beta, m)}{\beta},
$$

with $C(\beta, m)$ a universal dimensionless function. Defining $x=m \beta$, we can write it as a single variable function $C(x)$. At criticality it is proportional to the CFT central charge, $C(0)=-\pi c / 6[10,11]$. One can readily calculate the $1 \mathrm{D}$ energy

$$
\frac{E}{L}=-\frac{\partial \ln Z / L}{\partial \beta}=e_{0}-\frac{1}{\beta^{2}}\left(C-\beta \frac{\partial C}{\partial \beta}\right) .
$$

From the energy we can compute the thermodynamic entropy

$$
\frac{S}{L}=\beta \frac{E-F}{L}=-2 \frac{C}{\beta}+\frac{\partial C}{\partial \beta}=\frac{\pi c}{3 \beta}+O(1) .
$$

The specific ground state energy $e_{0}$ does not contribute to the entropy, which vanishes in the ground state, in accord with the third law of thermodynamics. At the $\mathrm{CP} S / L=\pi c / 3 \beta$, which is reminiscent of the relation between geometric entropy for a CFT and central charge found in [3].

The theorem of increase of the relative entropy (3) holds in general on a finite geometry and guarantees that $S_{\text {rel }}(\lambda, \beta)$ increases with $\lambda$ or, alternatively, with $m \propto \lambda^{1 / y}$. At the CP the theory is conformal invariant and $\Theta(z)=0$; hence $\lambda \partial S_{\text {rel }} / \partial \lambda=(m / y) \partial S_{\text {rel }} / \partial m=0$. Therefore, we propose to define an off-critical "central charge"

$$
C(x)=\beta^{2} S_{\text {rel }}(m, \beta),
$$

which is monotonic with $x$ and plays a similar role to Zamolodchikov's $c$ function. Thus we can express the monotonicity theorem in terms of dimensionless quantities simply as

$$
x \frac{d C}{d x}=\frac{\beta^{2}}{y} \int d^{2} z\langle[\Theta(z)-\langle\Theta(0)\rangle][\Theta(0)-\langle\Theta(0)\rangle]\rangle .
$$


While this form resembles that of Zamolodchikov, it is not quite the same. The correlator of $\Theta$ 's in the second term appears integrated. Furthermore, a detailed calculation of Zamolodchikov's function $c(m)$ for the free boson or fermion shows that it differs from $C(m)=$ $\left.C(x)\right|_{\beta=1}$. The cause is actually geometrical: A crucial step in the proof of Zamolodchikov's theorem relies on the assumption of rotation symmetry [2], which does not exist on the cylinder. Hence, one cannot obtain the theorem for it, contrary to the assertion in Ref. [8]. However, the absence of rotation symmetry is traded for the appearance of a new parameter, the length $\beta$, which can be used to obtain the monotonicity theorem above.

Besides, we may consider the behavior of the absolute 1D quantum entropy $S$ with respect to $\beta$ :

$$
\frac{\partial S}{\partial \beta}=\frac{\partial}{\partial \beta}(\beta E-\beta F)=\beta \frac{\partial E}{\partial \beta}=\beta \frac{\partial^{2}(\beta F)}{\partial \beta^{2}} .
$$

We have again monotonicity, for $\beta F$ is a convex function of $\beta$, as deduced from the expression of its second derivative as the average $-\left\langle(H-\langle H\rangle)^{2}\right\rangle$ with $H$ the total Hamiltonian, that is, including the kinetic term, unlike the monotonicity in [1]. This monotonicity is in principle unrelated with the monotonicity of $S_{\text {rel }}$ with respect to $m$. It allows us to define another monotonic dimensionless function, $\tilde{C}(x)=S / L m$. At the CP $S / L=\pi c / 3 \beta$, implying that $\tilde{C}(x)$ diverges at $x=0$, whereas $C(0)=0$. On the other hand, in the IR zone, $x \gg 1, C$ diverges as $C(x) \sim x^{2}$, whereas $\tilde{C}(x)$ decays exponentially.

We illustrate the form of finite-size corrections again with solvable models. For the Gaussian model the correction to the free energy can be expressed as the free energy of an ideal Bose gas,

$$
\beta \frac{F}{L}=e_{0} \beta+\int_{-\infty}^{\infty} \frac{d p}{2 \pi} \ln \left(1-e^{-\beta \epsilon(p)}\right),
$$

where the one-particle energy is $\epsilon(p)=\sqrt{p^{2}+m^{2}}$. This formula can also be obtained by an explicit calculation of the finite-size corrections [6]. When $m=0$ it can be used to calculate the central charge [11]. However, an expansion in powers of $m$ is not advisable: The ensuing integral at the next order is IR divergent; that is to say, the expression (18) is nonanalytic at $m=0$. Fortunately, the integral can be computed by changing the integration variable to $\epsilon$ and expanding the logarithm in powers of $e^{-\beta \epsilon}$. One obtains

$$
\beta \frac{F}{L}=e_{0} \beta-\frac{m}{\pi} \sum_{n=1}^{\infty} \frac{1}{n} K_{1}(n m \beta),
$$

where $K_{1}(x)$ is a modified Bessel function of the second kind. For large $x=m \beta / 2 \pi$ the correction is exponentially negligible but a small- $x$ expansion yields

$$
\begin{aligned}
\frac{C(x)}{2 \pi}= & -\frac{\zeta(2)}{2 \pi^{2}}+\frac{x}{2}+\frac{x^{2}}{2}\left(\ln \frac{x}{2}+\gamma-\frac{1}{2}\right) \\
& +\sum_{l=2}^{\infty}\left(\begin{array}{c}
1 / 2 \\
l
\end{array}\right) x^{2 l} \zeta(2 l-1) .
\end{aligned}
$$

The first term $\zeta(2)=\pi^{2} / 6$ gives the usual critical part and central charge $c=1$. The specific entropy (14) is

$$
\frac{S}{L}=\frac{\pi}{3 \beta}-\frac{1}{2} m+\beta \frac{m^{2}}{4 \pi}+O\left(m^{4}\right) .
$$

For the Ising model we have instead an ideal Fermi gas,

$$
\begin{aligned}
\beta \frac{F}{L} & =e_{0} \beta-\int_{-\infty}^{\infty} \frac{d p}{2 \pi} \ln \left(1+e^{-\beta \epsilon(p)}\right) \\
& =e_{0} \beta+\frac{m}{\pi} \sum_{n=1}^{\infty} \frac{(-)^{n}}{n} K_{1}(n m \beta),
\end{aligned}
$$

where the one-particle spectrum close to the CP is again $\epsilon(p)=\sqrt{p^{2}+m^{2}}$ and the integral is computed like the bosonic one. The small- $x$ expansion yields

$$
\begin{aligned}
\frac{C(x)}{2 \pi}= & -\frac{\zeta(2)}{4 \pi^{2}}-\frac{x^{2}}{2}\left(\ln \frac{x}{2}+\gamma-\frac{1}{2}\right) \\
& +\sum_{l=2}^{\infty}\left(\begin{array}{c}
1 / 2 \\
l
\end{array}\right) x^{2 l}\left(1-2^{2 l-1}\right) \zeta(2 l-1)
\end{aligned}
$$

Now the specific entropy is

$$
\frac{S}{L}=\frac{\pi}{6 \beta}-\beta \frac{m^{2}}{4 \pi}+O\left(m^{4}\right) .
$$

On the cylinder, the relative entropy $S_{\text {rel }}(\lambda, \beta)$ includes a finite-size contribution from $C$ but in general differs from the 1D absolute entropy $S$. Let us see if there is a relation between them for solvable models. We calculate the relative entropy for $W=-\ln Z /(\beta L)=F / L$. For the Gaussian model,

$$
\begin{aligned}
S_{\text {rel }}(r, \beta) & =W(r, \beta)-W(0, \beta)-r \frac{\partial W(r, \beta)}{\partial r} \\
& =S_{\text {rel }}(r)+\frac{1}{\beta^{2}}\left(C-C(0)-r \frac{\partial C}{\partial r}\right) \\
& =\frac{r}{8 \pi}-\frac{S}{2 L \beta}+\frac{\pi}{6 \beta^{2}} .
\end{aligned}
$$

For the Ising model the relative entropy is related instead to the $1 \mathrm{D}$ energy. Thus only for the Gaussian model $C$ and $\tilde{C}$ are closely related. In any event, for both Gaussian and Ising models it is easy to derive series expansions of $C$ or $\tilde{C}$.

The components of the stress tensor can also be calculated exactly for free models. Defining $\Theta:=T_{a}^{a}$ and $T:=T_{11}-T_{22}-2 i T_{12}$ we obtain [12]

$$
\begin{aligned}
& \langle\Theta(0)\rangle= \pm \frac{m^{2}}{2 \pi}\left(K_{0}(0)+2 \sum_{n=1}^{\infty}( \pm)^{n} K_{0}(n m \beta)\right), \\
& \langle T(0)\rangle= \pm \frac{m^{2}}{2 \pi}\left(K_{2}(0)+2 \sum_{n=1}^{\infty}( \pm)^{n} K_{2}(n m \beta)\right),
\end{aligned}
$$

with the same sign convention as before. The modified Bessel functions are divergent at zero, namely, $K_{0}(0)$ is logarithmic divergent and $K_{2}(0)$ is quadratically divergent. These are UV divergences, like the $\Lambda$ divergent terms of $W$. 
Using the recursion relations satisfied by the Bessel functions we can write the free energy (19) or (22) as

$$
\begin{aligned}
\beta \frac{F}{L} & =e_{0} \beta \mp \frac{m^{2} \beta}{2 \pi} \sum_{n=1}^{\infty}( \pm)^{n}\left[K_{2}(n m \beta)-K_{0}(n m \beta)\right] \\
& =-\frac{\beta}{2}\langle T(0)-\Theta(0)\rangle=\beta\left\langle T_{22}(0)\right\rangle
\end{aligned}
$$

showing its relation with the expectation values of the components of the stress tensor, which generalizes off the $\mathrm{CP}$ the standard relation [10]. Notice that it implies a definite form for $e_{0}$, namely, $e_{0}= \pm \frac{m^{2}}{4 \pi}\left[K_{0}(0)-K_{2}(0)\right]$, to be compared with (9).

Similarly, we can calculate

$$
\begin{aligned}
\frac{\partial W}{\partial r} & =\frac{\partial e_{0}}{\partial r} \pm \frac{1}{2 \pi} \sum_{n=1}^{\infty}( \pm)^{n} K_{0}(n m \beta)=\frac{1}{2 r}\langle\Theta(0)\rangle, \\
\frac{E}{L} & =e_{0} \pm \frac{m^{2}}{2 \pi} \sum_{n=1}^{\infty}( \pm)^{n}\left[K_{2}(n m \beta)+K_{0}(n m \beta)\right] \\
& =\frac{1}{2}\langle T(0)+\Theta(0)\rangle=\left\langle T_{11}(0)\right\rangle .
\end{aligned}
$$

The first equation is just a particular case of the expression of the derivative of $W$ with respect to $r$ as the expectation value of the "crossover part" of the action [1], since $\Theta$ is proportional to it. Furthermore,

$$
\frac{S(r, \beta)}{\beta L}= \pm \frac{r}{\pi} \sum_{n=1}^{\infty}( \pm)^{n} K_{2}(n m \beta)=\langle T(0)\rangle .
$$

Notice that this off-critical equation relates $\langle T\rangle$ with the entropy and therefore disagrees with the surmise in Ref. [5], which relates it with the free energy.

The relations between thermodynamic quantities and the expectation values of components of the stress tensor obtained above are not restricted to free-field models. To see this one must regard those expectation values as the response of $W$ to various geometrical transformations [10]; namely, a dilation in the $x_{2}$ direction, $\delta_{L} F=$ $(F / L) \delta L$ is given by $T_{22}$, a dilation in the $x_{1}$ direction, $\delta_{\beta}(\beta F)=E \delta \beta$ is given by $T_{11}$, a dilation in both directions, equivalent to a change of $r$, is given by $\Theta$, and a dilation in one direction plus a contraction in the other (shear transformation) is given by $T$, corresponding to $S /(L \beta)=\left\langle T_{11}\right\rangle-\left\langle T_{22}\right\rangle=\langle T\rangle$. However, closed expressions for these quantities can be obtained only for free models. For interacting models one can compute the finite size quantities above with conformal perturbation theory or the thermodynamic Bethe ansatz [13].

In conclusion, the $2 \mathrm{D}$ relative entropy grows with the coupling, according to a general theorem [1], which in 2D can be expressed in terms of the correlator of $\Theta$ 's, similarly to Zamolodchikov's theorem. Nevertheless, the function $C$ extracted from the relative entropy is not obviously connected with Zamolodchikov's $c$ function. On the other hand, the quantum 1D entropy satisfies a different monotonicity theorem with respect to $\beta$ or, say, the temperature, of pure thermodynamic nature, which leads to another monotonic function, $\tilde{C}=(\beta / m)\langle T\rangle$. The former function is well defined in the UV region whereas the latter is well defined in the IR region. As has been remarked before, a function monotonic with the RG is not unique [14]. Unlike Zamolodchikov's $c$ function, the quantities $C$ or $\tilde{C}$ have a clear physical origin. Therefore, the entropic monotonicity theorems proposed here constitute an interesting alternative formulation.

Let us make two comments on possible generalizations. A different approach uses the Wilson RG [15]. It is customary to try to prove the monotonicity theorem for the free energy. However, we believe that in that approach it is also some entropy the appropriate monotonic function, according to arguments presented previously [1]. Finally, the results in this paper can be generalized to higher dimensions $(d=3$ or 4$)$ and we expect them to contribute to the efforts to find a higher dimensional version of Zamolodchikov's $c$ theorem.

I acknowledge hospitality at the Nuclear Research Institute of Dubna (Russia), where this work was started, and partial support under Grant No. PB96-0887. I thank D. O'Connor for early conversations and M. A. R. Osorio for conversations on finite temperature QFT.

[1] J. Gaite and D. O’Connor, Phys. Rev. D 54, 5163 (1996).

[2] A. B. Zamolodchikov, JETP Lett. 43, 730 (1986).

[3] C. Holzhey, F. Larsen, and F. Wilzek, Nucl. Phys. B424, 443 (1994).

[4] D. Boyanovsky and R. Holman, Phys. Rev. D 40, 1964 (1989).

[5] N. E. Mavromatos and J. L. Miramontes, Phys. Lett. B 226, 291 (1989).

[6] C. Nash and D. O'Connor, Phys. Rev. Lett. 76, 1196 (1996).

[7] A. H. Castro Neto and E. Fradkin, Nucl. Phys. B400, 525 (1993).

[8] Maxim Zabzine, Report No. USITP-97-05, hep-th/ 9705015.

[9] In conformal perturbation theory the presence of logarithmic corrections is due to the existence of resonances; see Al. B. Zamolodchikov, Nucl. Phys. B348, 619 (1991).

[10] H. W. J. Blöte, J. L. Cardy, and M. P. Nightingale, Phys. Rev. Lett. 56, 742 (1986).

[11] I. Affleck, Phys. Rev. Lett. 56, 746 (1986).

[12] Computed with the methods of finite temperature QFT; see, e.g., H. W. Braden, Phys. Rev. D 25, 1028 (1982).

[13] J. Gaite (to be published).

[14] A. Cappelli, D. Friedan, and J. I. Latorre, Nucl. Phys. B352, 616 (1991).

[15] P.E. Haagensen, Y. Kubyshin, J. I. Latorre, and E. Moreno, Phys. Lett. B 323, 330 (1994); Vipul Periwal, Mod. Phys. Lett. A 10, 1543 (1995); J. Generowicz, C. Harvey-Fros, and T.R. Morris, Phys. Lett. B 407, 27 (1997). 